\title{
Neuroprotective effects of hypothermia and levetiracetam after hypoxia-ischemia in the neonatal mouse brain
}

\author{
Katja Straßer*, Daniela Brait, Laura Lückemann, Barbara Simone Reinboth, Josephine Herz, Ivo Bendix, \\ Ursula Felderhoff-Müser
}

From 50th Workshop for Pediatric Research

Gottingen, Germany. 20-21 March 2014

\begin{abstract}
Aims
Hypoxic-ischemic injury (HI) to the developing brain remains a major cause of morbidity. Hypothermia is currently the only established neuroprotective treatment available for term borns with hypoxic-ischemic encephalopathy, saving one in eight infants from developing severe neurological deficits. Therefore, additional treatments with clinically applicable drugs are indispensable. Furthermore, the pathophysiological mechanisms of hypothermia-induced recovery are not clearly understood. This study examines a potential additive neuroprotective effect of hypothermia combined with levetiracetam in neonatal mouse HI.
\end{abstract}

\section{Methods}

9-days-old C57BL/6-mice were subjected either to a shamoperation or to HI (modified Rice-Vannucci-model). After $\mathrm{HI}$, the pups were randomized into six groups: 1 ) no treatment, 2) hypothermia (whole body-cooling, 4 hours, $32^{\circ}$ C), 3) high-dose levetiracetam intraperitoneal $(70 \mathrm{mg} / \mathrm{kg}$ body weight), 4) hypothermia combined with high-dose levetiracetam intraperitoneal, 5) low-dose levetiracetam intraperitoneal (7 $\mathrm{mg} / \mathrm{kg}$ body weight), 6) hypothermia combined with low-dose levetiracetam intraperitoneal. Parameters of apoptosis (cleaved Caspase-3, TUNEL) and myelination (myelin basic protein) were analyzed 24 hours after $\mathrm{HI}$ by protein analysis and immunhistochemistry. From P28 to P60, cognitive and sensorimotor function was assessed via different tests.

\section{Results}

Hypothermia only and combined with low-dose levetiracetam was associated with a decrease of apoptosis and an increase of myelinated cells, but without additive effects. Intraperitoneal treatment with high-dose levetiracetam caused an increase of apoptotic factors. Behavioural testing demonstrated improved cognitive and sensorimotor outcome after treatment with hypothermia.

\section{Conclusion}

Whole-body cooling provides neuroprotection in the neonatal mouse brain by reducing apoptosis and preservation of myelination. However, treatment with levetiracetam after hypoxic-ischemic injury has no additive effects.

Published: 11 September 2014

doi:10.1186/2194-7791-1-S1-A7

Cite this article as: Straßer et al:: Neuroprotective effects of hypothermia and levetiracetam after hypoxia-ischemia in the neonatal mouse brain. Molecular and Cellular Pediatrics 2014 1(Suppl 1):A7. 